\title{
Modelling expertise for selecting manufacturing technology
}

\author{
Z. Zdráhal \\ Knowledge Media Institute, The Open University \\ Milton Keynes, $U K$, \\ phone +44 1908 65451, e-mail: Z.Zdrahal@open.ac.uk

\section{Valásek, J. Cermák} \\ Faculty of Mechanical Eng., Czech Technical University \\ Czech Republic, phone +4202 24357361, \\ e-mail:valasek@fsik.cvut.cz,cermak@fsid.cvut.cz
}

\begin{abstract}
In this paper we describe the development of a knowledge based system for selecting the technology for manufacturing machine parts. The problem solving method is modelled by instantiating of two standard generic task models - Heuristic Classification and Systematic Refinement - in the domain of bulk metal forming.
\end{abstract}

\section{Keywords}

Knowledge modelling, Generic Task Model, bulk metal forming, heuristic classification, systematic refinement

\section{INTRODUCTION}

In this paper we describe the development of a knowledge based system for selecting the specific technology needed for manufacturing machine parts. Our approach is based on constructing models of the problem solving activities and corresponding domain knowledge typically used for solving the problem. Based on the philosophy of Generic Task Models (GTM) (Tansley \& Hayball, 1993) we select the most appropriate model for the problem and refine it in the context of the technology selection problem. In addition, the GTM is used to drive knowledge acquisition and to organize domain knowledge into reusable ontologies. The knowledge models (i.e., GTMs), problem solving knowledge and domain 
knowledge are represented in the Operational Conceptual Modelling Language (OCML) developed at the Open University. The advantage of OCML is its operational semantics which makes it possible to execute the algorithm represented by the model. Based on the model a computationally efficient algorithm is implemented in Common Lisp. Although the problem solution requires reasoning about geometrical shapes we do not use a computational approach based on a formal representation of shapes. We prefer the user to assess the shapes and support his or her reasoning by integrating the knowledge based system with the graphical interface for shape classification.

The paper is organized as follows: in section 2 we introduce the problem of selecting manufacturing technology. In section 3 we briefly describe the knowledge modelling techniques used in paper and the knowledge modelling tools we used for developing the application. In section 4 we instantiate and refine the knowledge models for the problem of selecting manufacturing technology. In section 5 we describe domain ontologies and show the graphical interface for reasoning about shapes. In the concluding section we summarise the lessons learned from this application. The work presented in this paper has been done as a pilot application of the Encode project (Copernicus 94-0149) funded by the CEC under the Copernicus programme.

\section{SELECTING MANUFACTURING TECHNOLOGY}

The problem we address in this paper is the one solved by the technologist when preparing the technological documentation for manufacturing a new product. The product is specified by a technical drawing accompanied by other technical documentation which prescribes additional product characteristics, such as required tolerances, the material it is to be manufactured from, the number of products to be manufactured (lot size), expected batch size etc. The available manufacturing technologies are characterised at the general level by their typical properties and individually, for each manufacturer, by the technical data for available machine tools. The technologist's task is to find a cost-effective technology for manufacturing the product and specify the technology by a sequence of technological operations which can be carried out on the available machine tools.

As we will show later, manufacturing technologies can be organized into a hierarchy of technology types. The lower in the hierarchy the more details of the technology are specified. In this paper we will demonstrate our approach by considering only bulk forming technologies. However, this restriction does not decrease the general applicability of our approach; it only means that the initial decisions in favour of bulk forming have already been made outside of our system. Even with this limited scope, the selection of the cost-optimal bulk forming technology is a very complex and knowledge intensive problem. There are many types of constraints which define the solution space. These include relations specifying that the technology is be able to produce the shape from the prescribed 
material in the required precision; economical criteria in the form of soft constraints and preferences; associations of technologies, machine tools and technological operations the machines can carry out; and forces and energies the machine tools can produce.

Decision-making is based on heuristic knowledge supported by the calculation of additional technological parameters. To solve the problem we need knowledge of two domains: we must understand the potential of different technologies and we must be able to recognize and evaluate important characteristics of the manufactured product. The initial knowledge analysis of this problem has been described in (Kucera et al., 1995). In the following sections we will show how the knowledge acquired from domain experts can be modelled and the models assembled to form a complete method for technology selection.

\section{KNOWLEDGE MODELLING METHODS TOOLS}

In his seminal paper Newell postulates the existence of a knowledge level which comprises the knowledge needed for solving complex problems but is independent of implementation details (Newell, 1982). Knowledge intensive information processing systems can be viewed at the knowledge level in terms of high level concepts such as tasks and their structures, problems solving methods and their associated knowledge roles which allow us to model the expertise needed for solving the problem. We consider the knowledge-level models as primary concepts. The domain expertise can be viewed as an instantiation of the model integrated with expert's knowledge. The expert centred view of early expert systems has been therefore replaced by a more structured, model-based approach. The models of expertise without the domain specific knowledge can be reused for solving problems of a similar type. In accord with the KADS terminology (Tansley \& Hayball, 1993) we call these domain-independent models Generic Task Models (GTM), see also (Chandrasekaran, 1986). GTMs specify typical problem solving scenarios, the inferences to be made and the knowledge structures needed to enable these inferences.

New KBS applications can be developed by selecting and refining a GTM in the context of the problem. Domain knowledge, such as the hierarchy of technologies or attributes of the product, is described as separate domain models independently of the problem solving method. These models are later used to supply the GTM with the required domain context. On the other hand, the choice of a GTM which solves the problem also defines the knowledge needed for the GTM and therefore GTMs can be used to drive knowledge acquisition. Unambiguous definitions of the concepts upon which the model depends are essential for reusing knowledge models. The formal conceptualizations of the universe of discourse are usually called ontologies.

We describe knowledge models as ontologies represented in the Operational Conceptual Modelling Language (OCML). OCML is a language defined on top of 
Common Lisp (Motta, 1996) and consists of an object oriented system, a backward chaining theorem prover (Prolog) and a forward chaining inference system (OPS5). In OCML ontologies can inherit definitions from other ontologies. This mechanism allows the new model to reuse already existing concepts. Knowledge level concepts are defined in terms of OCML primitives: classes, instances, relations and functions. For example, a task is a class with input and output slots, and a goal slot describing the relation which must the task upon completion satisfy. A problem solving method is a task with additional slots specifying task decomposition into subtasks and the corresponding control flow. Primitive tasks are not solved by task decomposition, instead the problem solving method is a functional specification of inference steps. GTMs are tasks and therefore can be represented in OCML and inherit properties of OCML tasks. Since GTMs do not refer to any specific domain, they define the structure of the required knowledge and not by the content. We will call such knowledge placeholders knowledge roles. The actual domain concepts and their relations are described in OCML as domain ontologies. The mapping from domain ontologies to knowledge roles is defined as an OCML relation and represented, for example, by a set of mapping rules.

Building knowledge models is supported by graphic editor Ousel (Watt, 1996) which allows the user to visualize knowledge models in terms of the most frequently used primitives such as tasks, roles, classes, objects and relations. Figures 1, 3 and 4 in this paper are screen snapshots of Ousel models. Ousel graphical representations can be translated into OCML code and, vice versa, OCML models can be downloaded to Ousel, providing that they use OCML primitives which have graphical counterparts in Ousel.

\section{SELECTING AND INSTANTIATING GTM}

As we have indicated above the applicable manufacturing technology is selected by comparing the required parameters of the product with the manufacturing capabilities of individual technologies. From the manufacturing viewpoint products can be grouped in accordance with different criteria, such as shape, material, accuracy, lot size etc. We consider as primary the grouping of products according to their shapes. The group is a class of objects characterised by the same generalized shape properties. The reason for selecting the shape as a primary criterion is that the products of the same shape group can be usually manufactured by the same type of technology. Specific features of individual products from the same shape group such as shape details, prescribed accuracy or production lot size allow us to refine the technology type selected for the shape class and work out as detailed specification of technology as necessary for manufacturing.

This model of technologist's reasoning best fits the Heuristic Classification GTM. The "horse shoe" model of Heuristic Classification is well known for its use as a model of diagnostic reasoning in Mycin. By choosing the same GTM we are saying that at the abstract level the reasoning process of assigning the manufacturing 
technology to a product is isomorphic with assigning the therapy to a patient though the domain knowledge is complete different. The instantiation of the Heuristic Classification GTM for the problem of selecting manufacturing technology is shown in Figure 1.



Figure 1. The Ousel Workbench and the Heuristic Classification GTM.

The left-hand part of the model i.e. the classification of products into shape groups is conceptually simple but technically very difficult because it requires reasoning about shapes from the geometry of objects represented by technical drawings. The select-shape-class task in Figure 1, can be more easily and more accurately solved by the user though the computer may support and verify user's decisions. Our system presents the user with definitions of shape classes, assists in selecting one and verifies whether the object could be in principle associated with the selected class. Shape classes are arranged into a hierarchy shown in Figure 2.

Each shape class corresponds to a single cell of the table. The first level in the shape hierarchy consists of basic shapes represented by rows in the table and associated with conditions which all objects of that basic shape class must satisfy. Basic shapes are further divided by more specific shape descriptors into columns. The graphical interface allows the user to browse shape tables until he or she finds the shape class which best characterizes the object on the technical drawing. To assist the user with class selection there are three shape descriptors presented for each class: (1) a verbal shape characterization, (2) conditions associated with characteristic dimensions of basic shapes, and (3) examples of typical objects belonging to the class. 


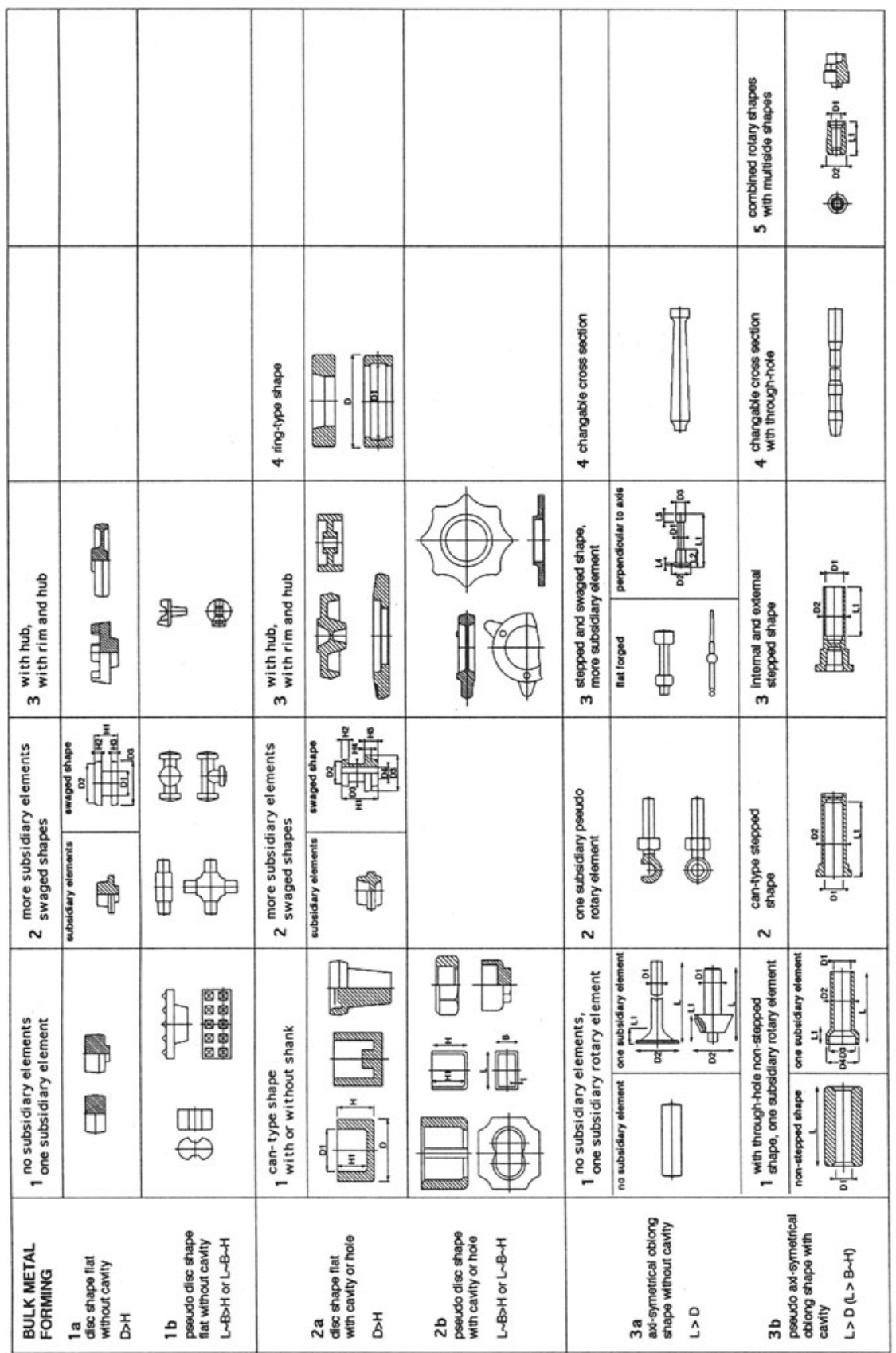

Figure 2. Hierarchy of shape classes. 
The user compares these descriptors with the object in the drawing and selects the class by clicking on the corresponding cell of the table and inputs the characteristic dimensions associated with the shape class. These values are used for evaluating the shape class criterion. The shape table interface in Figure 2 has associated shape ontology represented in OCML (shape classes are OCML classes and conditions are OCML relations). Each shape class in the ontology has an associated technology types which can be used for manufacturing objects of this shape. This assignment is the heuristic knowledge used by the assign-technology task shown in Figure 1.

The task specialize-technology shown in Figure 1 models the process of refining the specification of the selected technology. Specialisation is guided by product data and by the relationship between product data, technologies and available machine tools. The product data describe required accuracy, product weight, material, lot size etc. The relationship between product data and manufacturing technologies is a part of the domain expertise and includes criteria for evaluating the cost-effectiveness of the technology. This domain knowledge is represented as a number of OCML domain ontologies (accuracy ontology, weight classification ontology, lot size ontology etc). In addition, the hierarchy of manufacturing technologies is represented as an OCML ontology. The relevant part of the hierarchy is shown in Figure 3 as displayed by the Ousel graphical editor.

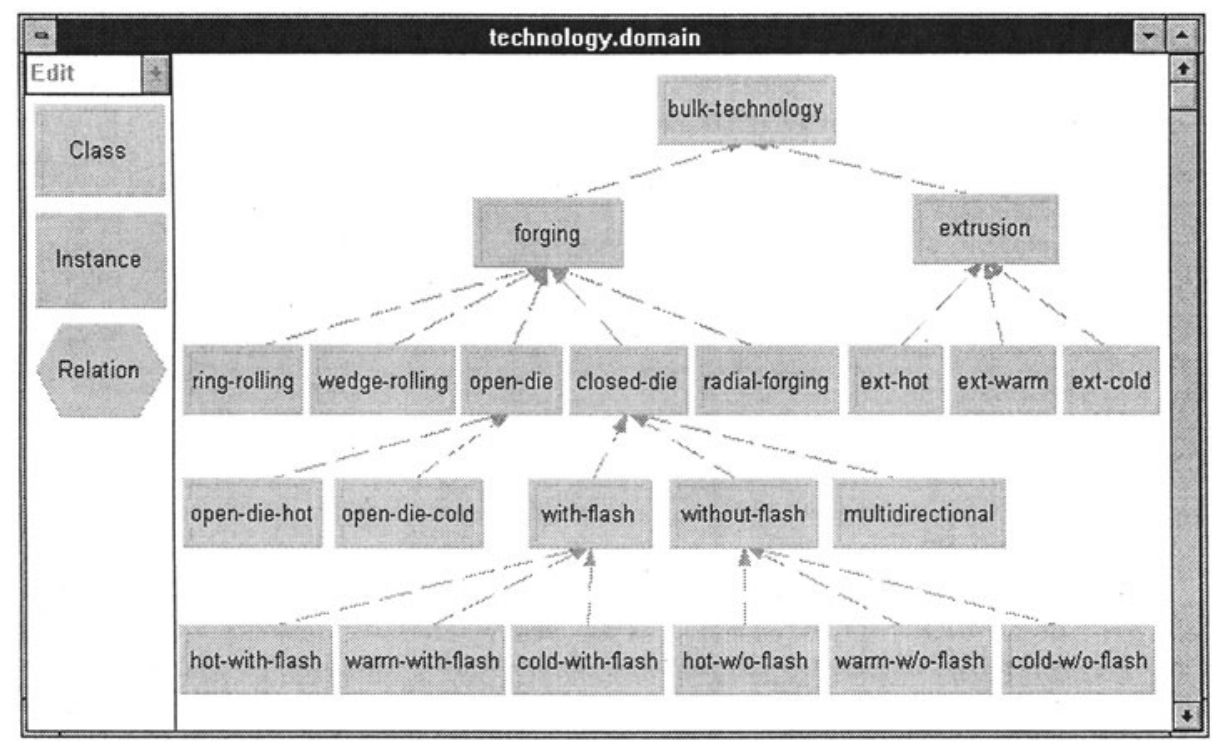

Figure 3. Hierarchy of bulk forming technologies

Before analysing the technology refinement task in the next section let us recapitulate the modelling decision taken so far as shown in Figure 1. The product shape described by a technical drawing is first generalised into a shape class. A 
heuristic association assigns to the shape class a set of applicable technology types which are then specialised by using product requirements to complete their detailed specification. The first inference step involves traversing the shape hierarchy upwards towards more general shapes, the third inference step consists of traversing the technology hierarchy downwards towards fully specified technologies. The abstraction from the technical drawing to shape classes is done by the user, the correspondence between shape classes and technology types is a simple heuristic association between shape classes and technology types. In the next section we will model and analyse the task called specialize-technology in Figure 1. In particular, we will show part of the task modelled using Heuristic Classification can be modelled in detail using another GTM.

\section{GTM FOR REFINING SELECTED TECHNOLOGY}

We will describe the specialize-technology task by modelling its internal structure. Systematic Refinement (Tansley \& Hayball, 1993) is the most appropriate GTM for refining the details of the selected technology. The data flow diagram of this GTM is shown in Figure 4. Systematic Refinement is suitable if the object description is characterised by a hierarchical is- $a$ structure. The object in our case is the manufacturing technology which indeed is hierarchically organized, see Figure 3.

Systematic Refinement has two input roles: the object description and object attributes. In the context of our problem the object description and object attributes correspond to technology-type and product-attributes, respectively - see also the notation in Figure 4. Systematic Refinement specifies details of an object description by comparing attributes observed in the object with those expected by the hierarchy. This method faithfully models the technological reasoning described in the previous section.

There are six tasks in Systematic Refinement we need to define. The first one, select-hierarchy, assigns an is-a hierarchy to the object description. For the technology selection problem this task is trivial, the only existing hierarchy is the one shown in Figure 3. The decompose task selects a more refined technology as the next hypothesis. Tasks select-value-p and select-value-t are similar. They select value of an attribute from the corresponding attribute set: select-value-p from the attributes associated with the product; select-value-t, the value associated with the hypothesized technology in the hierarchy. In terms of technological concepts selectvalue-p selects prescribed characteristics of the product while select-value-t finds out what the technology which is being assessed can provide.

The compare task compares the prescribed characteristics of the product with those provided by the current hypothesis. If the prescribed parameters cannot be achieved, the technology is rejected, otherwise the technology remains as a candidate for further refinement. 


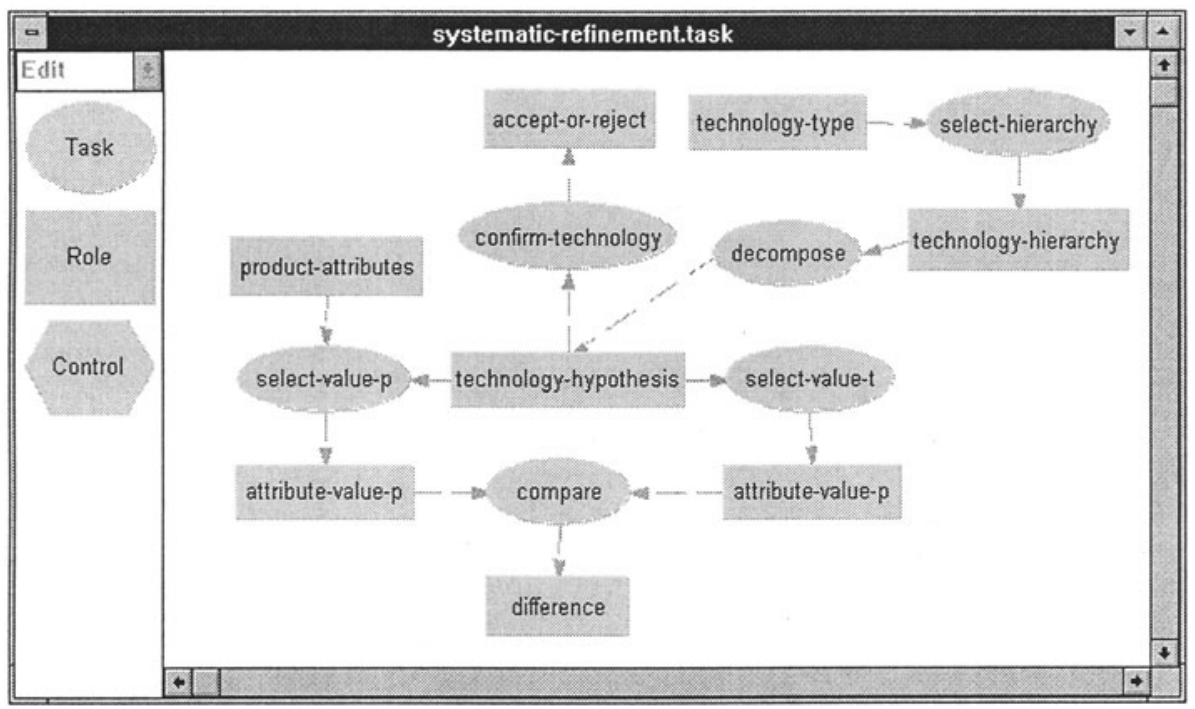

Figure 4. Refining selected technology type by the Systematic Refinement GTM.

The last task in Figure 4 is confirm-technology which simply verifies whether the technology has been already completely specified, i.e. whether the search has reached a leaf node of the hierarchical tree.

\section{MODELLING KNOWLEDGE FOR REFINING TECHNOLOGY}

The product attributes we consider for deciding on additional technological details are either directly available from the product specification - i.e., from a technical drawing and product description, or require additional computation and reasoning. A typical example of the attribute which can be found in the product description is the required product accuracy. We express the accuracy classes in terms of the Tolerance grade ISO (IT). The lower IT grade the smaller tolerance is allowed i.e., the greater the precision required of the product. The IT grade of the product can be used directly to eliminate some technologies. For example, the technologies which process a hot work-piece achieve in general lower shape accuracy. We model this knowledge as an OCML relation mapping the IT accuracy classes to the technology hierarchy. The product material, weight and the lot size play similar role in technology refinement. The order in which these attributes are chosen may affect the efficiency of search but we ignore this fact because the search space is too small and moreover, the initial analysis of the domain did not provide any useful search-pruning heuristics.

For further refinement of applicable technologies the class shapes shown in Figure 2 are too coarse-grained. It is necessary to take into consideration a measure of 
shape complexity. This is defined by means of an envelope body of the product which is an notional object of cylindrical or rectangular shape circumscribing the product. Each shape class in the shape table has assigned one or more possible envelope bodies since for some product shapes it is not clear in advance whether the envelope body should be a cylinder or a rectangular shape. The shape complexity coefficient is then calculated from the smallest one. For example, shape class $2 \mathrm{~b}-1$ in Figure 2 is associated with rectangular and cylindrical envelope bodies. The user estimates the dimensions of all applicable envelope bodies and the system makes the choice. The shape complexity coefficient is estimated as a ratio of the volume of the product and the volume of the selected envelope body. The volume of the product is calculated from its weight and specific density of the material. However, for very unusual shapes the ratio may not express the intuitive concept of shape complexity. Therefore, the user is asked to approve the shape complexity coefficient or to correct its value based on his or her professional judgement.

Shape complexity is an example of an attribute whose value requires additional calculation and user reasoning. However, in the Systematic Refinement method it is used in a similar way as directly observable attributes. The calculation of attribute values could be modelled as another task. For the sake of brevity these models are not presented in this paper.

The final attribute used for technology refinement is based on evaluating available machine tools. Each machine tool is characterised by technologies it can be used for and by its parameters. The most important parameters we have included in the knowledge model based on Systematic Refinement GTM are maximum forces and deformation energies produced by the machine. These values must be higher than the corresponding values needed for forming the product. Their product related values are calculated from the shape, weight and material parameters. By considering the machine tool related parameters we assign machines to the technology.

\section{CONCLUSIONS}

The system described in this paper has limitations, which stem from the very idea of specifying the technology for manufacturing by selecting from a set of existing, already known technologies. In the case of technological innovations the manufacturing technology is not selected but designed or invented. Obviously, in such a case our model cannot be applied. We assume that more complex models based on open-ended generic design GTMs might be applicable.

The second limitation is a consequence of our way of handling uncertain knowledge. In our ontologies we consider only crisp relations describing compatibility criteria between technologies and the product attributes. However, in reality these relation should be modelled as fuzzy relations since the compatibility 
is usually described as a "measure of suitability". In the current solution we have made this simplification to avoid additional complication with a fuzzy calculus.

The described experiment had a number of goals, which were satisfied. They include:

- Testing the knowledge modelling methodology on the problem of a selection type. We have had an extensive experience with using this approach to solve design problems (Zdrahal \& Motta, 1995). In (Motta \& Zdrahal, 1998) we have elucidated the relationship between knowledge level concepts and weak, symbol level problem solving techniques. The initial problem analysis presented in (Kucera et al, 1995) assumed weak problem solving methods and had not been not guided by any specific knowledge model. The question was whether the benefit of imposing additional GTM structure on the existing knowledge would outweigh the effort. The experiment proved that we could easily identify typical reasoning processes in the existing knowledge and consequently the selection of GTM's was not too difficult. Since the technology selection is a real world, relatively complex problem with many dependencies among different types of knowledge we assumed that the knowledge analysis would also generate redundant and possibly inconsistent knowledge. This proved to be true. By associating problem solving knowledge with the knowledge roles of the GTM we have identified and removed these redundancies and inconsistencies. The systematic model-based methodology has made knowledge maintenance and update much easier in comparison with our earlier systems solving similar tasks which were developed by rapid prototyping (Kopecky et al., 1985). The experiment also showed how knowledge models can be built by selecting and assembling component models from a model library.

- As part of the current Encode project and the previous Vital project we have developed a set of knowledge modelling tools. The experiment served as a test of their applicability to real problems and therefore generated very useful feedback for their improvement.

- A significant result of the knowledge modelling experiment is the developed system itself, which although it can solve typical cases, needs more extensive testing before it can be used in the industrial practice.

The system development produced another important result: The knowledge modelling exercise had a significant impact on the way one of the co-authors (J. Cermák) - the domain expert involved in the development - has reorganised and restructured the knowledge he presents in the courses on metal forming offered at the Faculty of Mechanical Engineering of the Czech Technical University. We assume that the system with additional browsing facilities will be available to students, either as a software running locally or over the Web. 


\section{REFERENCES}

Cermák J., and Valásek M. (1998). Knowledge Based Selection of Metal Forming Technology. Accepted for the International Conference on Metal Forming, Birmingham, April 1998.

Chandrasekaran B. (1986). Generic tasks in knowledge-based reasoning: High-level building blocks for expert system design. IEEE Expert 1. No. 3. pp. 23-30.

Kopecky P., Lazansky J., Marík V. and Zdráhal Z. (1985). Knowledge-Based System for Computer Aided Process Planning. Proceedings of the IFAC Conference. Beijing, China. pp. 245-251.

Kucera F., Král M., Cermák J., and Valasek M. (1995). Analysis of Manufacturability (Analysis of Techniques for Selecting Manufacturing Technology). Encode Technical Report, (Project Copernicus). Faculty of Mechanical Engineering, Czech Technical University. Prague.

Motta E. (1996). Knowledge-level modelling in OCML. Course material. DM871: Intelligent Systems: Analysis and Design. The Open University.

Motta E. and Zdrahal Z. (1998). An approach to the organization of a library of problem solving methods which integrates the search paradigm with task and method ontologies. Accepted for publication in the International Journal for Human Computer Studies.

Newell A. (1982). The Knowledge Level. Artificial Intelligence. Vol. 18, No. 1. pp. 87127.

Tansley D.S.W. and Hayball C.C. (1993). Knowledge-Based Systems Analysis and Design - A KADS Developer's Handbook. Prentice Hall.

Watt S. (1996). Knowledge-level modelling in Ousel. Course material. DM871: Intelligent Systems: Analysis and Design. The Open University.

Zdráhal Z. and Motta E. (1995). An In-Depth Analysis of Propose \& Revise Problem Solving Methods. In "Proceedings of the 3rd Japanese Knowledge Acquisition for Knowledge-Based Systems Workshop, JKAW '94" (R.Mizoguchi, H.Motoda, J.Boose, B.R.Gaines and P.Compton eds.). pp. 89-105.

\section{BIOGRAPHY}

Z. Zdráhal is a Senior Research Fellow at the Knowledge Media Institute, The Open University, UK. His research interests include knowledge modelling, casebased reasoning and sharing knowledge over the Web.

M. Valásek is a Professor of Mechanics at the Faculty of Mechanical Engineering, Czech Technical University. He is interested in computational mechanics of multibody systems, mechatronics and knowledge based support for engineering design.

J. Cermák is an Associated Professor of Manufacturing at the Faculty of Mechanical Engineering, Czech Technical University. He is interested in bulk metal forming technology, computational methods for bulk forming technology. 